\title{
Instrumentation Requirements for Automatic Power Quality Analysis and Dissemination
}

\author{
Espírito Santo, António e Calado, Maria do Rosário \\ Dep. Engenharia Electromecânica - Universidade da Beira Interior \\ 6201-001 Covilhã - Portugal \\ aes@demnet.ubi.pt
}

tel.:+351-275-329758, fax: $+351-275-329972$

\section{Introduction}

Nowadays, electrical energy quality in power systems is a major concerning problem [1], and must be monitored and analyzed. In the recent past, the power quality supplied to an electrical system wasn't a priority, and only had reflection in system reability. Recently, the scenario tends to be changing and is necessary to identify, process, save and disseminate, preferentially in an automated way, the anomalies, causes and origins. This is the only way to involve all entities within the electrical energy sector, working in co-operation to achieve the required power quality.

In the past, few applications were sensitive to power supply's disturbances. Nowadays, because of the electronic equipment proliferation, it is necessary to execute a rigorous control of the energy quality supplied to the processes.

The great majority of the equipments that includes microprocessed components, or any other kind of electronic regulation and command, are sensitive to power supply disturbances. However, they are also responsible for the energy quality deterioration supplied to other devices sharing the same installation. This circumstance can, in a limit situation, provoke disturbances in neighboring plants.

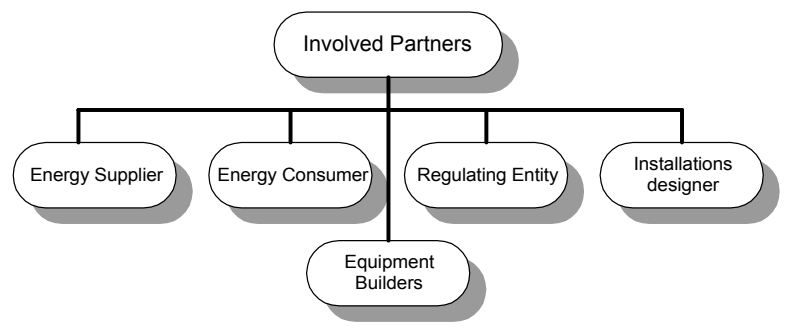

Fig. 1 - Entities involved in the electrical energy quality process.

The increasing integration of productive systems allows an inoperativeness or even inefficacy of the whole system in case of a failure on one of its components. This may happen if there is not adequate redundancy of the various functions. That is the reason why nowadays one can compare the perturbation effects on a system feeding to the effects of a power failure in the past, with all the consequences normally associated on the quality and production domain.

So, we must identify all entities interested in make a deep study of the electrical power quality problem (Fig. 1), and find efficient mechanisms to observe and diagnose the disturbances.

\section{Potential Involved Entities}

The energy providers are obliged by contract to give to the customer a raised quality service. To respect this, they must possess efficient ways to identify and quantify the disturbances, was well as the responsible. With this information, the energy provider can react efficiently, either taking corrective measures, or inserting penalties in the consumer energy bill. The energy provider can also offer a service in this intervention domain.

The customer expects a high quality energy product, in accordance with the contract celebrated with the supplier, allowing him to develop its activities in a competitive way. For beyond the normal requirements in quality terms, as they are the maximum duration of the supply interruptions or maintenance of lines, today customers want to see assured, from the supplier, a set of others necessities. These demands require perfect knowledge, in real time, of the installation and what happens inside it. The customer must know the impact that the quality energy deterioration will have on him [2]. For this, he must be able to evaluate the impact on its businesses, or, in a general way, in its organization. Every time the equipment has its performance affected, the consumer must possess data, which confronted with the one given by the deliverer, obligates the last one to assume the responsibility over the verified losses. Also, the adoption of efficient solutions techniques will be based on concrete information.

Connecting the two last entities, supplier and customer, we have a third entity, which assumes the role of regulation entity. Beyond the traditional obligations, 
this organization must also be worried in supervise the energy product quality and defend the undefended consumers rights, as in case of domestic consumers. This role can be carried out by the municipal authority, or municipal authority associations, in the scope of activities associated to the promotion of the quality in the cities that they represent. In the industrial consumer's case, producers associations would carry out the regulation task.

Electric installations designer is directly interested in that problem, and cooperates closely either with the distributor, or with the energy consumer. An appropriate knowledge of disturbances that might penalize more the future plant user, as well as the eventual disturbances that might be present on it, also are added value to this interposer. The installation designer can, with this knowledge, adopt technical solutions that minimize the side effects from potential distributions, making a judicious choice for the installation topology, and correctly select protection devices.

The equipment builder is another one interested in the knowledge of the disturbances that can affect equipments built by him. Many times, the problem is, in fact, on the electrical installation and not obligatorily on the equipment. It is exactly in this field that all the other partners can have an active role, informing the builders on the real conditions that their equipments must support. They should even go further more to the point of defining tests that the equipments manufacturers should realize, in order to verify the capabilities that their equipments have to tolerate expected disturbances.

\section{Energy perturbations classification}

There are many different definitions for power quality perturbations [3],[4]. A short reference will be done next.

\section{A. Transient}

In accordance with standard ANSI C62.41-1991 while the voltage transient has a 50 microseconds typical duration, the current transient last typically 20 microseconds.

A transient impulse normally is characterized by its rise and decay times. Being able to present a positive or negative polarity, meaning that it can add or subtract to the voltage or current signal. The transient impulses can excite the natural frequency of the energy system and produce transient oscillations.

Transient oscillations are expressed has a disturbance of voltage, current, or both, from the stationary regimen with double polarity, where the value of is magnitude decay with time. This event can be described by its frequency contents, allowing the identification of predominant frequency.

This transient phenomenon can be promoted by thunderstorms, capacitor banks switching (Fig. 2), or electronics loads commutations. The transient impulses have origin, mainly, by the presence of large loads applied to electrical motors, in the loss of one of the phases, or improper ground connection.
Transient events can cause immediate destruction, or early failure, of equipment controlled by microprocessor. For more sensitive equipment, it can occur situations where its performance is compromised.

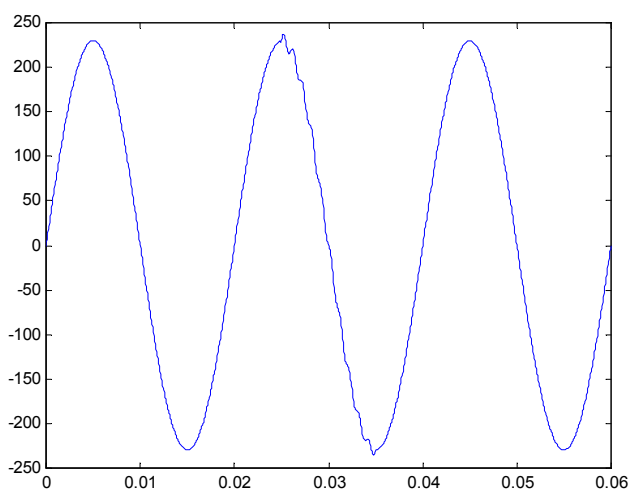

Fig. 2 - Transient oscillation.

\section{B. Long duration variations}

A long duration variation always occurs when electrical signals magnitude limits are violated for periods longer than one minute. Standard ANSI C84.1 1982 specifies tolerances of electrical power systems.

Long duration variations can assume the overvoltage form as result of switching operations (disconnect large loads or connecting capacitors banks), or also assume the undervoltage form resulting of from inverse events, associated with that produced overvoltages. This phenomenon is not promoted by systems malfunctions, but by dynamic systems variations or switching operations.

If the value of voltage is null, for a period longer than one minute, the long duration voltage variation is considered as a supported interruption. This kind of occurrence mostly requires human intervention so that service normality can be restituted.

\section{Short duration variation}

The short duration voltage variations can have origin in the occurrence of faults, turning of large loads, or existence of deficient transmission lines connections.

Depending on localization, origin, and kind of installation, the perturbation can promote temporary sags, swells, or complete voltage interruption.

A sudden reduction in RMS voltage (sag voltage), or current, with duration longer than half cycle and shorter than one minute, can turn off the load, or take it to an unstable function regimen (Fig. 3). This happens, normally, with electronic equipment, when supplied voltage goes below $20 \%$ of rated value. The main sources, associated with this phenomenon, are large motors starting, sudden load increases, faults in distribution lines, or power electronics that consumes current pulses. This class of defects can have origin inside or outside the installation, and can represent about $60 \%$ of the energy quality problems.

According to standard ANSI 1100-1992 swells phenomenon are surge voltages, or currents, at the rated 
energy system frequency, for periods that go beyond half cycle to some seconds, and can be originated by events internal or external to the installation. The phenomenon can occur with loss of one of the three power lines, with a serious load decrease, or a reload after an energy failure. Swells are the less frequent disturbances, representing only 2 to $3 \%$ of all the problems in industry. The overvoltage arises, normally, from motors faults, lighting systems, and electronic malfunctions. In electronic controlled equipment serious appliance damages can occur when only 6 to $10 \%$ overvoltage is applied.

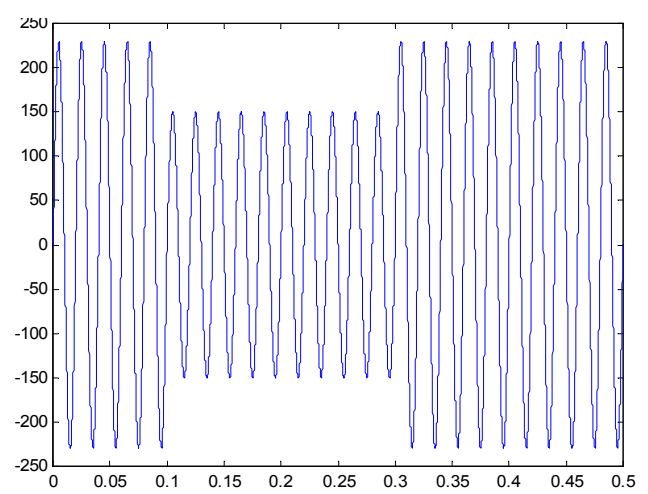

Fig. 3 - Short duration perturbation.

\section{Voltage unbalance}

Voltage unbalance is defined as corresponding to the maximum deviation from three-phase voltages, or currents, average, and is expressed in percentage, or can be defined using symmetrical components. The relation between positive and negative sequences can be used to specify the unbalance percentage. This situation can be due to single-phase load supplied by three-phase system, or, when fault protection acts only in one phase.

\section{E. Waveform deformation}

Waveform deformation can be defined as a steady deviation from the sinusoidal shape, and can be characterized by its spectral content. Use of dimmer technologies in incandescence light bulbs, implemented with diodes, can be one of the origins. When DC component is present in the electrical power system, a premature saturation in the transforming magnetic circuits is verified, with the corresponding loss of performance level. The grounding system is also affected by corrosion, promoted by electrolyze phenomenon that occur when DC current is present.

Noise is defined as an undesirable electronic signal, with a high spectral content, which overlaps system voltage, or current. Noise can have his origin in electronic power devices, control circuits, solid state rectifiers, and PC voltage sources.

Notching phenomenon derives from the normal operation of power electronic devices, and is associated with the occurrence of commutation between phases. During this event, a short circuit between phases is verified, decreasing voltage values depending on system impedance for the observation point.

\section{F. Voltage fluctuations}

Standard ANSI C84.1-1982 specifies voltage fluctuation as a systematic, or random, envelope variation of voltage signal, which magnitude normally does not exceed the limits.

Loads demanding fast current variations can be responsible by the flicker phenomenon. This term has origin in the visual impact promoted in the incandescent illumination light bulbs when low frequency fluctuations are present.

\section{G. Variations in the nominal frequency}

The variation in frequency is measured in terms of maximum acceptable deviation from rated value. In most energy systems this type of occurrence is very rare. Only in presence of a serious energy system fault, involving a large geographical area, one can observe this type of disturbance. This phenomenon is more problematic when isolated generators are used, and the control does not possess enough robustness to become insensitive to load disturbances.

\section{Mathematical tools for power quality analysis}

The information contained in a signal cannot be so perceivable at the time domain as it is at the frequency domain. So, signal detection and characterization can therefore be made with more effectiveness with frequency domain analysis. The Fourier Transformation (FT) analytical expression from the signal $x(t)$ is given by

$$
X(f)=\int_{-\infty}^{+\infty} x(t) \cdot e^{-2 j \pi f t} d t
$$

and the inverse transformation is obtained applying the inverse Fourier Transformation

$$
x(t)=\int_{-\infty}^{+\infty} X(f) \cdot e^{2 j \pi f t} d f .
$$

In the previous expressions, $t$ represents time, and $f$ the frequency. The FT discloses the frequency content from a signal in the time domain, but cannot give any kind of information about the moment in time where these frequencies occurred. This information only makes sense when the signal is stationary in time domain. This is not the situation in the majority of the energy disturbances presented previously. In order to solve this problem one can assume that in short time slices, the signal keeps stationary. If these pieces are considered small enough, this is the same as observed the signal through a small time window. From this point of view, the signal is exactly stationary. This methodology was the origin to the STFT (Short Time Fourier Transformation).

Only a small difference exists between the STFT and the FT. That is, the signal is seccionated in pieces small enough so that it can be considered stationary. On this purpose a window $W$ is selected, having a length small enough so the stationary condition is verified. If the window amplitude is the unit, then the section returned is identical, but another type of windows can be used. The 
TF is carried out through the optioned signal after the window $W$ application. The following step will shift the window for a new location and repeat the previous process. This procedure follows until the end of signal is reached. The following analytical expression summarizes the process

$$
\operatorname{STFT}_{X}^{\omega}(t, f)=\int_{t} x(t) \cdot \omega^{*}\left(t-t^{\prime}\right) \cdot e^{-j 2 \pi f t} d t
$$

Where $x(t)$ is the signal in analysis, and $W(t)$ the window function. As can be observed this transformation is identical to the FT, only the window is shifted in time domain.

The STFT problem has some similarities with the uncertainty of Heisenberg. In a simple manner, and in analogy with the moment and the localization of one particle in movement, this principle affirms that one cannot know in simultaneously the exact representation of a signal both in time and frequency domain. What one can know are the time periods in which some band frequencies exist. This property depends on window length. Remember that in the FT the frequency resolution is not a worrying problem, because an infinite length window is used, in contrast, there isn't time resolution. On the other hand, in the STFT the window length is finite in time, covering only one portion of the signal. This reduces the frequency resolution, meaning that is no more possible to know exactly what frequencies are present in signal. One only knows the frequency band containing the exact frequency.

It is possible to make a signal study using an alternative approach, with Multiresolution Analysis (MRA) [8]. In this case, the signal is analyzed at different frequencies with different resolutions. Each spectral component receives now a different treatment, contrarily to the STFT case. The MRA provides good temporal resolution and low frequency resolution at high signal frequencies, and good frequency resolution with low time resolution at low signal frequencies. This approach makes all sense, if one thinks that longer perturbations possess lower frequency contents, and shorter perturbations possess higher frequency contents [6],[7].

The Wavelet Transformation (WT) [5], was developed as an alternative to the STFT. This transformation procedure is identical to the STFT. The signal is convoluted by a function in similarity to the window at the STFT. However, The length of the window is modified with the computation process, being the main characteristic of the WT. The continuous definition of the WT is given by

$$
C W T(\tau, s)=\frac{1}{\sqrt{|s|}} \int x(t) \psi^{*}\left(\frac{t-\tau}{s}\right) d t
$$

Has can be seen in (4), the signal returned by the transformation is a two variables function $(t, s)$, where the translation function $\psi(t)$ is called the mother wavelet.

\section{Automatic system topology}

The system topology can be composed by three different components (Fig. 4). The Monitor units will be spread through the installation, and will detect all perturbation. After perturbation detection, the Monitor unit will send it to the Concentration unit, that receives information from Monitor units, and elaborates automatically the reports destined to all partners involved in the process. The last component will be the Client unit, that receives and format the reports send to him through the Concentration unit.

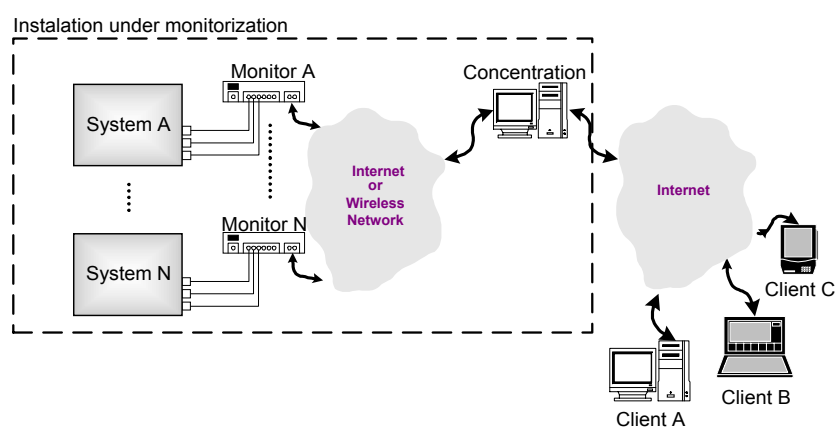

Fig. 4 - Automatic system monitor for power quality observation and dissemination.

\section{A. Monitor unit}

Knowing that power quality analysis can be included in digital signal processing, and taking in to account that Digital Signal Processors (DSP) are extremely accessible at low cost, justifies the development of a Monitor unit based on it. These machines have special architecture characteristics [9],[10], required to power quality processing. As an example, one can refer the Analogue to Digital Converter (ADC), Timers and Compare Units that can be used to synchronize the acquisition process. The Harvard architecture employs independent buses to memory and program access. This allows simultaneous write and read operations in data and program spaces.

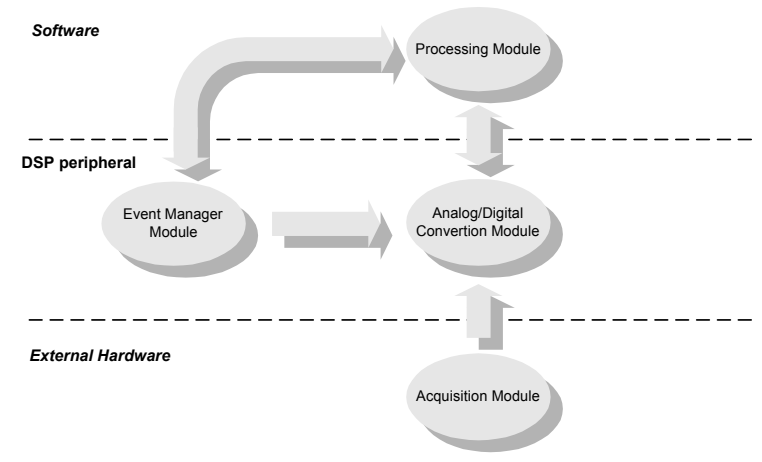

Fig. 5 - Monitor unit topology.

DSP have also an instruction set designed to speed up signal processing algorithms, helped by high-speed arithmetic units consisting of adders and multipliers which can be used in parallel.

A power quality monitoring system must carry out the following tasks:

1. Acquire all voltage and currents

2. Save equipment status.

3. Detect all perturbations and inform automatically the Concentration unit.

4. Receive from the Concentration unit new parameterization data. 
Three different layers compose the Monitor unit topology (Fig. 5): external hardware, DSP peripheral devices, and DSP software.

One can identify the Acquisition Module, who makes signals levels accommodations to the Analogue/Digital Conversion Module, controlled by the Event Manager Module based in timer's DSP.

The Event Manager Module produces interruptions with the periodic pattern observed in Fig. 6. The three first interruptions correspond to voltage and current acquisition on different channels (three-phase systems). The last interruption is devoted to processing the detection algorithm. This interruption pattern repeats itself in time, with a frequency imposed by the detection algorithm processing needs.

The Processing Module is a software piece, and realizes different tasks. All system management is made in a foreground/background kernel. Priority tasks like detection algorithms, on the fly modules configurations, and systems management occur at the foreground plane. The background plane is used to establish communication with the Concentration unit.

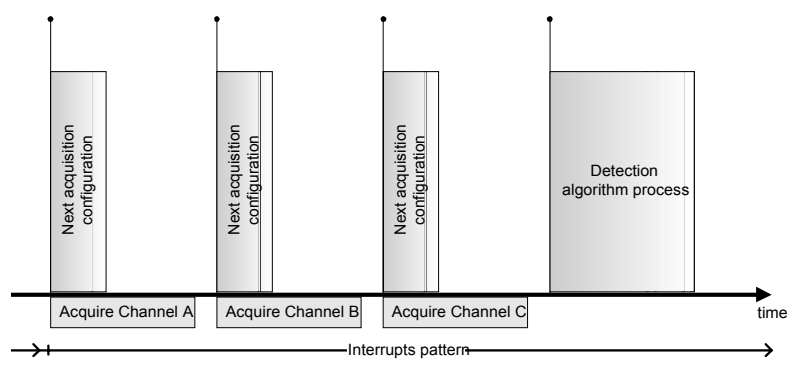

Fig. 6 - Sequential pattern produced by the Event Manager Module.

\section{B. Concentration unit and Client units}

The information obtained by the Monitor unit must be sent to the Concentration unit. Internet is the most powerful way to make the connection between all system components. Also exists evident advantages in using a wireless network to connect the Monitor units and the Concentration unit.

Plants without an efficient monitoring system depends strongly from human intervention. Time needed to inform the decision center that a failure had occurred is inevitable, and critical either for the equipment or for the production. Also, one must consider the time needed to make the correct decision and put it on practice.

Owning measurement instrumentation is insufficient. It is also necessary to count with help from highly specialized technicians, who aren't always available, and moreover are very expensive. The automatic perturbation detection, identification and resolution are essentially systematic tasks. The Concentration unit brings another advantage by the introduction of some kind of artificial intelligence [11] for elaboration of different reports based in the needs of each client. Expert systems could be a solution [12]. This computer program can solve problems using knowledge representation with inference techniques. Other interesting solutions will be the introduction of methodologies based in neural-fuzzy or genetic algorithms.

The Concentration unit must be universally supported, so the Java programming language, with Java Virtual Machine (JVM), is the perfect tool to develop an Internet based application. Java also allows that various parts of the automatic perturbation detection, identification, and resolution program run on different machines.

Using the Clients units all entities can access to Concentration unit trough web browser. Reports and alarms can be remotely configured in conformity with the entities needs.

\section{Conclusions}

This article introduced a set of outstanding details that one must take into account to develop an automatic system monitor. Monitor system characteristics are both conditioned by the user necessities, and kind of perturbation that needs to be observed.

With this concerning in mind, one has to identify the involved entities and their particular characteristics and needs. A short perturbation classification was also made, opening the way for discussion of different mathematical possible tools used to detect and study perturbations. The functional topology proposed tries to satisfy all needs. With this tool all entities will be able to develop a cooperative work, trying to achieve the required power quality.

Some of the described features, like the Monitor unit, are in the phase of implementation. The next steps go in the direction of developing the Concentration unit, including on it all the characteristics drafted in this paper.

\section{References}

[1] Coelho, D. A., Matias, J. C. O. e Espírito, A. E. "Extending Certification to Energy Management Systems: A Contribution to Improve Energy Efficiency in Industry", ECEEE 2003 Summer Study, Côte d'Azur, 2-7 June 2003.

[2] Espírito Santo, A. Matias, J., "Qualidade Energética no Processo Produtivo". Engenharia '2003 - Inovação e Desenvolvimento. Universidade da Beira Interior, Covilhã, Novembro 2003.

[3] Santoso, Surya., Electrical Power Systems Quality. McGraw-Hill Professional; 2nd edition (November 26, 2002).

[4] Dugan, Roger C., Electrical Power Systems Quality, McGraw-Hill, 1996.

[5] Mallat, Stephane G. A Wavelet Tour of Signal Processing. Academic Press, 1999.

[6] X. Xu, M. Kezunovic. "Automated Feature Extraction from Power System Transients Using Wavelet Transform". 
[7] G.T.Heydt and A.W.Galli, "Transient power quality problems analyzed using wavelets," IEEE Trans. Power Delivery, vol. 12, April 1997.

[8] Polikar R., “The Wavelet Tutorial”, http://users.rowan.edu/ polikar/WAVELETS/WTt utorial.html.

[9] TMS320F/C240 DSP Controllers Reference Guide - CPU and Instruction Set, Texas Instruments, 1999.

[10] TMS320F/C240 DSP Controllers Reference Guide - Peripheral Library and Specific Devices, Texas Instruments, 1999.

[11] M. M. Morcos, W.R. Anis Ibrahim, "Electric Power Quality and Artificial Intelligence: Overview and Applicability", International Signal Processing Conference, March31-April3, 2003, Dallas, Texas.

[12] Peter Jackson, Introduction to Expert Systems, Addison-Wesly, Third Edition, 1998. 